\title{
Application of Molecular Dynamics and Monte-Carlo Methods near the Critical Points
}

\author{
Balabaev N.K., Lakhno V.D. \\ Institute of Mathematical Problems of Biology RAS, Keldysh Institute of Applied Mathematics \\ of Russian Academy of Sciences, Pushchino, Russia
}

Abstract. The applicability of molecular dynamics and Monte-Carlo methods near the phase transition is discussed on the example of DNA melting.

Key words: statistical sum, characteristic scale, denaturation, heat capacity, Landau-Lifshitz theorem.

Molecular dynamics (MD) and Monte Carlo (MC) methods are a powerful tool for solving modern problems of modeling atomic-molecular systems. If the interactions between individual atoms and molecules are considered to be known, then the MD and MC methods can replace a full-scale experiment. The MD and MK methods, however, have different application areas. Formally, the MD method is more general, since it contains the MC results as its limiting case, which is reduced to the study of statistical equilibrium. It is believed that the MD results in this limit should be compared with the "exact" MC result.

A direct comparison of the numerical calculations of the thermodynamic characteristics by the MD and MC methods demonstrates that the simulation results coincide exactly far away from the phase transition point. Near this point, however, such a comparison turns out to be invalid. The reason for the discrepancy between the MD and MC results near the phase transition point is as follows. All real potentials of interaction between atoms and molecules are finite functions of the distances between them. Hence it follows that the corresponding statistical sum (configuration integral), if no special cutoff is introduced, diverges. Far away from the critical point, for example, at a temperature much lower than the dissociation temperature of the molecule, the introduction of a cutoff does not change the result. This is due to the fact that the number of trajectories leading to an infinite contribution to the statistical sum of the system is very small. In practical calculations this contribution can be formally eliminated in various ways. For example, it is possible to establish a potential wall at large distances in the interaction potential. Another way is to simply discard the trajectories going to infinity in the statistical averaging. Finally, one can also straightforwardly count the entire ensemble of particles, then the average distance between particles in the ensemble will be the characteristic cutoff scale. At the critical point per se, however, there is a complete arbitrariness in the choice of the characteristic scale. The MC method turns out to be inapplicable in this case, since it turns out to be entirely due to the choice of the characteristic scale.

Formally, the MD method allows simulating any dynamic processes. However, when modeling a phase transition in an equilibrium system, in order to obtain an adequate result, an infinite time for calculating the trajectories and the number of realizations is required, which makes its direct application impossible.

Let us illustrate this with an actual example of DNA melting simulation performed in [1, 2]. The most important result of these works was that the results of simulating DNA melting by the MC and MD methods completely coincide to the right and to the left of the phase transition point, far from it. This is easy to see by comparing, for example, the curves of the heat capacity, obtained by the method [1,2], with the MC results for this system in [3]. Near the phase 
transition point, the results of MD and MC are different. Naturally, the question arises which of the results obtained by these two methods is closer to reality (under the assumption that the PBD model used in $[1,2]$ corresponds to reality). As follows from the above analysis, at the phase transition point per se, the MC method, although it deals with an equilibrium state, gives an arbitrary result, which entirely depends on how the simulation is carried out.

At the same time, the MD method is unable to calculate the equilibrium state, but it is quite capable of calculating quasi-equilibrium. In a real experiment, we are always dealing not with equilibrium, but with quasi-equilibrium. Therefore, it is these calculations that reflect reality and their compatibility with experiment.

Let us summarize briefly the above statements. In case [3], the MC method is used to consider true equilibrium in a system with an infinite potential wall. The MD method searches for a quasi-equilibrium state in the same model with the same wall, which, in principle, cannot be obtained by the MC method. Therefore, as applied to a real system, the MC method may give incorrect results and the MD method must be applied. Hence it follows that the theory of phase transitions is, generally speaking, inapplicable to quasi-equilibrium states. For example, in the case of first-order phase transitions, instead of point transitions in quasi-equilibrium systems, diffuse transitions will be observed, to which the standard classification of transitions may not be applicable.

For example, the Landau-Lifshitz theorem on the impossibility of phase transitions in a 1d system is well-known [4]. Note that this theorem refers to true thermodynamic equilibrium. As mentioned above, in reality, for potentials bounded at infinity corresponding to a real situation, we are dealing not with a true equilibrium, but with a quasi-equilibrium, to which the LandauLifshitz theorem is inapplicable.

\section{REFERENCES}

1. Likhachev I.V., Lakhno V.D. The direct investigation of DNA denaturation in PeyrardBishop-Dauxois model by molecular dynamics method. Chem. Phys. Lett. 2019. V. 727. P. 55-58. doi: 10.1016/j.cplett.2019.04.027

2. Likhachev I.V., Lakhno V.D. Investigation of DNA denaturation in Peyrard-BishopDauxois model by molecular dynamics method. Eur. Phys. J. B. 2019. V. 92. Article No. 253. doi: 10.1140/epjb/e2019-90741-6

3. Teplukhin A.V. Calculation of Heat Capacity of Peyrard-Bishop-DauxoisChains by Monte Carlo Method. In: Proceedings of the International Conference "Mathematical Biology and Bioinformatics". Ed. V.D. Lakhno. Vol. 8. Pushchino: IMPB RAS, 2020. Article No. e5. doi: $10.17537 / \mathrm{icmbb20.13}$

4. Landau L.D., Lifshitz E.M. Statistical Physics. Part 1. Moscow: Fizmat. lit, 1976. 